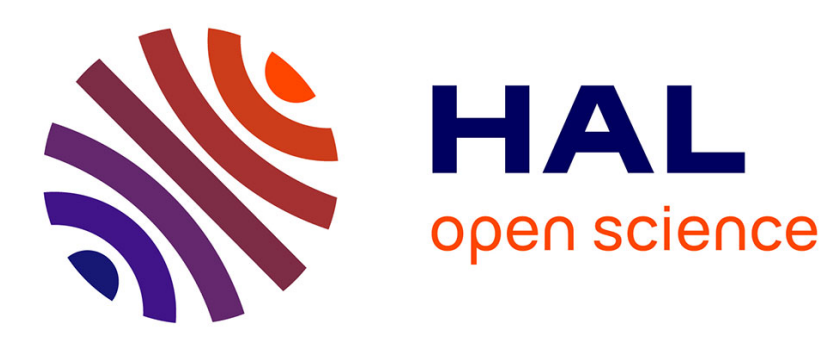

\title{
Nonlinear aspects of high Reynolds number channel flows
}

\author{
Patricia Cathalifaud, Jacques Mauss, Jean Cousteix
}

\section{To cite this version:}

Patricia Cathalifaud, Jacques Mauss, Jean Cousteix. Nonlinear aspects of high Reynolds number channel flows. European Journal of Mechanics - B/Fluids, 2010, vol. 29, pp. 295-304. 10.1016/j.euromechflu.2010.02.002 . hal-00745781

\section{HAL Id: hal-00745781 \\ https://hal.science/hal-00745781}

Submitted on 26 Oct 2012

HAL is a multi-disciplinary open access archive for the deposit and dissemination of scientific research documents, whether they are published or not. The documents may come from teaching and research institutions in France or abroad, or from public or private research centers.
L'archive ouverte pluridisciplinaire HAL, est destinée au dépôt et à la diffusion de documents scientifiques de niveau recherche, publiés ou non, émanant des établissements d'enseignement et de recherche français ou étrangers, des laboratoires publics ou privés. 


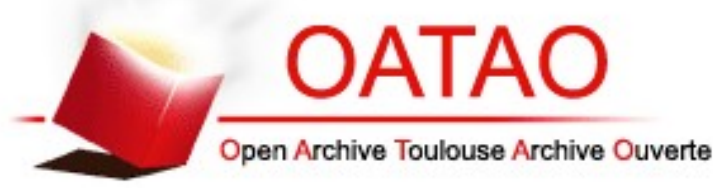

\section{Open Archive Toulouse Archive Ouverte (OATAO)}

OATAO is an open access repository that collects the work of Toulouse researchers and makes it freely available over the web where possible.

This is an author-deposited version published in: http://oatao.univ-toulouse.fr/ Eprints ID: 6325

To link to this article: http://dx.doi.org/10.1016/j.euromechflu.2010.02.002 URL: http://www.sciencedirect.com/science/article/pii/S0997754610000178

\section{To cite this version:}

Cathalifaud, Patricia and Mauss, Jacques and Cousteix, Jean Nonlinear aspects of high Reynolds number channel flows. (2010) European Journal of Mechanics - B/Fluids, vol. 29 ( $n^{\circ}$ 4). pp. 295-304. ISSN 0997-7546

Any correspondence concerning this service should be sent to the repository administrator: staff-oatao@listes.diff.inp-toulouse.fr 


\title{
Nonlinear aspects of high Reynolds number channel flows
}

\author{
P. Cathalifaud ${ }^{\mathrm{a}, \mathrm{b}, *}, \mathrm{~J}$. Mauss ${ }^{\mathrm{a}, \mathrm{b}}$, J. Cousteix $^{\mathrm{c}, \mathrm{d}}$ \\ a Université de Toulouse; INPT, UPS; IMFT (Institut de Mécanique des Fluides de Toulouse); Allée Camille Soula, F-31400 Toulouse, France \\ ${ }^{\mathrm{b}}$ CNRS; IMFT; F-31400 Toulouse, France \\ ${ }^{\text {c } D e ́ p a r t e m e n t ~ M o d e ̀ l e s ~ p o u r ~ l ' A e ́ r o d y n a m i q u e ~ e t ~ l ' e ́ n e r g e ́ t i q u e, ~ O N E R A, ~} 2$ avenue Édouard Belin, B.P. 4025, 31055 Toulouse Cedex 4, France \\ d Institut Supérieur de l'Aéronautique et de l'Espace, 10, avenue Édouard Belin, 31055 Toulouse Cedex, France
}

Keywords:

Interactive boundary layer

Separation

Channel flow

Asymptotic analysis

\begin{abstract}
A B S T R A C T
This paper considers the flow in a two-dimensional channel at high Reynolds number, with wall deformations which can lead to flow separation. An asymptotic model is proposed by using the successive complementary expansion method with generalized asymptotic expansions. In particular, the model emphasizes the asymmetry of the channel geometry by introducing a change of variables. It is shown that the model is more general than the models developed with the method of matched asymptotic expansions. Comparisons with Navier-Stokes solutions show that the model is well founded and enables us to treat original problems.
\end{abstract}

\section{Introduction}

We consider a plane Poiseuille flow in a two-dimensional channel perturbed by wall deformations - troughs or bumps which are sufficiently severe to induce flow separation.

At high Reynolds number, an asymptotic analysis can be applied. When the Reynolds number tends to infinity, the first step consists of a straightforward simplification of the Navier-Stokes equations leading to the Euler equations. So, it is tempting to call for a hierarchical process. In the vicinity of singular zones, near the walls, the second step consists of trying to correct the first approximation by a boundary layer analysis.

However, it is known that, in many problems involving a strong coupling, this type of hierarchical approach is not possible and the two approximations must be considered simultaneously. In fact, the solution of the inviscid flow equations depends on the Reynolds number and it is better to talk about Euler equations rather than inviscid flow equations as is done very often.

If we decide that the numerical solution of Navier-Stokes equations is not desirable, because either the numerical cost to find such a solution is too big, or a better understanding of physical phenomena is desired, a suitable approach consists of

\footnotetext{
* Corresponding author at: Université de Toulouse; INPT, UPS; IMFT (Institut de Mécanique des Fluides de Toulouse); Allée Camille Soula, F-31400 Toulouse, France.

E-mail addresses: catalifo@imft.fr, catalifo@cict.fr (P. Cathalifaud) mauss@cict.fr (J. Mauss), Jean.Cousteix@onecert.fr (J. Cousteix).
}

using the asymptotic model called the interactive boundary layer (IBL) model.

Then, we are faced with a paradox. The only constructive way to establish asymptotic approximations is to use a small parameter, here the inverse of the Reynolds number, which goes to zero. This leads to regular asymptotic expansions and to a hierarchical approach.

Excluding a multi-layer approach of triple deck type, which introduces very restrictive hypotheses on the scales, a possibility is to use generalized asymptotic expansions. According to this method, the small parameters of the problem can be included in the functions which form the expansions. This conception is very different because the small parameters are not considered as tending towards zero but are only small. This is easy to conceive if a uniformly valid approximation (UVA) is constructed from the standard method of matched asymptotic expansions.

In fact, the reasoning can be inverted by starting from an assumed form of the UVA. In order to extend the techniques on which the method of matched asymptotic expansions is based, the successive complementary expansion method (SCEM) has been developed [1]. When regular approximations are sufficient, it can be shown that the regular SCEM is equivalent to the method of matched asymptotic expansions associated to a modified Van Dyke matching principle.

Thanks to the generalized expansions, interaction effects between the Eulerian and boundary layer regions are considered simultaneously and the construction of a UVA does not require any matching principle; only the boundary conditions of the problem are used. 
In a channel, there is no external flow region and the asymptotic models for the flow perturbations are mainly based on an inviscid rotational core flow region together with boundary layers near the walls; a comprehensive discussion of this structure can be found in Sobey [2]. The asymptotic analysis of these flows has been performed essentially by Smith [3], and a systematic approach has been proposed later on by Cousteix and Mauss [4]. More recently, the modelling of channel flows has been examined by Lagrée et al. [5] and by Lagrée and Lorthois [6].

After a presentation of the SCEM (Section 2), the problem is formulated (Section 3), then the flow is analysed with SCEM using standard hypotheses on the wall deformations (Section 4). In Section 5, a new approach is proposed in order to emphasize the role of the asymmetry of the channel geometry. As shown in Section 6, the resulting model contains the standard model obtained by Smith $[7,8,3]$ but is more general. Finally, the numerical technique is presented in Section 7 and results are given with comparisons with Navier-Stokes solutions (Section 8).

\section{About the SCEM}

The purpose of this section is to give a short overview of the SCEM, which is explained in more detail in Cousteix and Mauss [1]. Definition of a generalized asymptotic approximation.

First of all we have to define clearly what is an asymptotic approximation of a function. Two functions $\Phi(x, \varepsilon)$ and $\Phi_{a}(x, \varepsilon)$ defined in a domain $D$ are asymptotically identical to order $\delta(\varepsilon)$ if their difference is asymptotically smaller than $\delta(\varepsilon)$, where $\delta$ is an order function:

$\Phi(x, \varepsilon)-\Phi_{a}(x, \varepsilon)=\mathrm{o}(\delta)$,

where $\varepsilon$ is a small parameter arising from the physical problem studied. The function $\Phi_{a}$ is called an asymptotic approximation of the function $\Phi$. The converse being also true, it must be stressed that the goal of an asymptotic approximation is to replace $\Phi$ by a simpler function $\Phi_{a}(x, \varepsilon)$. This simplicity comes from the different methods used to construct asymptotic expansions (AE), generally defined by

$\Phi_{a}(x, \varepsilon)=\sum_{i=0}^{n} \delta_{i}(\varepsilon) \varphi_{i}(x, \varepsilon)$,

where the asymptotic sequence of order functions $\delta_{i}$ is such that $\delta_{n} \succeq \delta$ and such that all functions $\varphi_{i}$ are of strict order $1, \varphi_{i}=$ $\mathrm{O}_{\mathrm{S}}(\overline{1})$. This is called a generalized asymptotic expansion because $\varepsilon$ is included in the developed functions.

Definition of a regular asymptotic approximation.

In many cases, it is possible to construct a regular $\mathrm{AE}$, with an asymptotic sequence noted $\delta_{i}^{(0)}$, which can be written

$\Phi_{a}(x, \varepsilon)=E_{0} \Phi=\sum_{i=0}^{n} \delta_{i}^{(0)}(\varepsilon) \varphi_{i}^{(0)}(x)$

For this reason, when the $\mathrm{AE}$ is not regular, it is called a generalized asymptotic expansion. Strictly, it is not necessary to add the qualifying adjective "generalized" but, too often, it is thought that an $\mathrm{AE}$ is necessarily regular. Such an asymptotic approximation is objectively simpler than the function $\Phi$ since the construction is made step by step by limiting processes. This is the reason why, following Eckhaus [9], we define, for a regular AE, a special operator $E_{0}$ called the "expansion operator" at a given order $\delta$; thus, $\Phi-E_{0} \Phi=0(\delta)$.

Method of matched asymptotic expansions (MMAE).

Interesting cases occur when the function $\Phi$ is not regular in $D$, i.e., when $\mathrm{AE}(2)$ or (3) of $\Phi$ is valid only in a restricted region $D_{0} \in$
$D$ called the outer region. We are faced with a singular perturbation problem and we must introduce boundary layer domains. Here, in the simplest case, we introduce an inner domain which can be formally denoted $D_{1}=D-D_{0}$ and located near the origin. The boundary layer variable or inner variable is $X=\frac{x}{\zeta(\varepsilon)}, \zeta(\varepsilon)$ being the order of thickness of this boundary layer. If a regular expansion can be constructed in $D_{1}$, we can write

$$
\Phi_{a}(x, \varepsilon)=E_{1} \Phi=\sum_{i=0}^{m} \delta_{i}^{(1)}(\varepsilon) \varphi_{i}^{(1)}(X) .
$$

This inner "expansion operator" $E_{1}$ is defined in $D_{1}$ at the same order $\delta$ as the outer expansion operator $E_{0}$; thus, $\Phi-E_{1} \Phi=\mathrm{o}(\delta)$. The links between these expansions $E_{0} \Phi$ and $E_{1} \Phi$ are the rules or heuristic principles which define the asymptotic matching.

To our knowledge, the best principle is the modified Van Dyke principle (MVDP), which can be found in [1] and can be written $E_{0} E_{1} \Phi=E_{1} E_{0} \Phi$. As the expansions are defined at the same order $\delta$, we can construct a composite approximation at this order, or an asymptotic approximation, which can be written

$\Phi_{a}=E_{0} \Phi+E_{1} \Phi-E_{1} E_{0} \Phi$.

This is clearly a uniformly valid approximation (UVA) defined at order $\delta$.

The successive complementary expansion method (SCEM).

For singular perturbation problems, the successive complementary expansion method (SCEM) is based on the knowledge of generalized asymptotic expansions and upon the idea that the reasoning using the method of matched asymptotic expansions (MMAE) must be inverted. First, the structure of the uniformly valid approximation (UVA) must be assumed and then the method to construct the UVA is deduced. This point of view has been adopted in other methods like WKB or multiple scale methods, but their usefulness is restricted to particular problems.

We assume that two significant subdomains have been identified, classically called the outer and inner domains. According to the SCEM, a UVA $\Phi_{a}$ of the function $\Phi$ is supposed to have the following structure:

$\Phi_{a}(x, X, \varepsilon)=\sum_{i=1}^{n} \bar{\delta}_{i}(\varepsilon)\left[\bar{\varphi}_{i}(x, \varepsilon)+\bar{\Psi}_{i}(X, \varepsilon)\right]$.

We say that $\Phi_{a}$ is a UVA of $\Phi$ defined at order $\bar{\delta}_{n}$ if $\Phi=$ $\Phi_{a}+\mathrm{o}\left(\bar{\delta}_{n}\right)$. Again, this UVA is called generalized because classical understanding used rather "regular" asymptotic expansion where $\varepsilon$ is not in the developed functions. For instance, we can write

$\Phi_{a}=\Phi_{a r}+\mathrm{o}\left(\delta_{m}\right)$,

where $\Phi_{a r}$ is a regular UVA such that $\bar{\delta}_{n}=\mathrm{O}\left(\delta_{m}\right)$, and

$\Phi_{a r}(x, X, \varepsilon)=\sum_{i=1}^{n} \delta_{i}(\varepsilon)\left[\varphi_{i}(x)+\Psi_{i}(X)\right]$.

The sequence of order functions $\bar{\delta}_{i}$ may or may not be the same as the sequence $\delta_{i}$ since the elements of this latter sequence are gauge functions. Recall that a gauge function is an a priori choice of an order function in its equivalence class.

The SCEM has already been used in its regular form [10,11], but not in its generalized form. Under the name of composite expansions, Nayfeh [12] discusses the related technique developed earlier.

In its regular form, the SCEM is equivalent to the MMAE with the modified form of the Van Dyke matching principle (MVDP). This is a crucial point since the matching is a consequence of the chosen form of the UVA and not the contrary. 
The ambiguous writing of the generalized SCEM needs guidelines to enable construction of a UVA. In general, knowledge of the physics of the problem, as will be seen later, provides us with an efficient solution.

One can be guided by the fact that if the solution $\Phi$ of integrodifferential equations is formally written $L_{\varepsilon} \Phi=0$, then, for the UVA given by (6) we have $L_{\varepsilon} \Phi_{a}=R_{n}(x, \varepsilon)$. Since we have assumed that the boundary conditions are exactly satisfied for $\Phi_{a}, R_{n}$ is small in a certain sense. This is ascertained by estimate theorems which can be obtained, in general, only for linear problems. The generalized form proposed here is very well adapted to strong interaction problems leading logically to the models of interactive boundary layers.

\section{Formulation of the problem}

The dimensionless Navier-Stokes equations are

$\frac{\partial \mathcal{U}}{\partial x}+\frac{\partial \mathcal{V}}{\partial y}=0$,

$L_{\varepsilon} U=U \frac{\partial U}{\partial x}+\mathcal{v} \frac{\partial U}{\partial y}+\frac{\partial \mathcal{P}}{\partial x}-\frac{1}{\mathcal{R}}\left(\frac{\partial^{2} u}{\partial x^{2}}+\frac{\partial^{2} u}{\partial y^{2}}\right)=0$,

$L_{\varepsilon} \mathcal{V}=u \frac{\partial \mathcal{V}}{\partial x}+\mathcal{V} \frac{\partial \mathcal{V}}{\partial y}+\frac{\partial \mathcal{P}}{\partial y}-\frac{1}{\mathcal{R}}\left(\frac{\partial^{2} \mathcal{V}}{\partial x^{2}}+\frac{\partial^{2} \mathcal{V}}{\partial y^{2}}\right)=0$

The above equations are written in an orthonormal axis system. Coordinates $x$ and $y$ are reduced by the width $W^{*}$ of the basic channel whose lower wall is $y=-\frac{1}{2}$ and upper wall is $y=$ $\frac{1}{2}$ (Fig. 1). The velocity components are reduced by a reference velocity $V^{*}$ defined below by (12). The Reynolds number is

$\mathcal{R}=\frac{\varrho^{*} V^{*} W^{*}}{\mu^{*}}$,

where $\varrho^{*}$ and $\mu^{*}$ are the dimensionalized density and viscosity coefficient.

The reference velocity $V^{*}$ is expressed as a function of the basic pressure gradient $\frac{\mathrm{d} p_{0}}{\mathrm{~d} x}$ or, equivalently, of the dimensionalized mass flow $Q^{*}$ per unit width of the channel:

$Q^{*}=\varrho^{*} V^{*} W^{*} \int_{-1 / 2}^{1 / 2} u_{0} \mathrm{~d} y, \quad V^{*}=6 \frac{Q^{*}}{\varrho^{*} W^{*}}$,

$\frac{\mathrm{d} p_{0}}{\mathrm{~d} x}=-\frac{2 \mu^{*}}{\varrho^{*} V^{*} W^{*}}$,

where the basic plane Poiseuille flow is

$u_{0}=\frac{1}{4}-y^{2}, \quad v_{0}=0, \quad p_{0}=-\frac{2 x}{\mathcal{R}}+p_{c}$,

and $p_{c}$ is an arbitrary constant.

The flow is perturbed by indentations of the lower and upper walls:

$y_{l}=-\frac{1}{2}+F(x, \varepsilon), \quad y_{u}=\frac{1}{2}-G(x, \varepsilon)$,

where $\varepsilon$ is a small parameter (Fig. 1).

At high Reynolds number, the reduced equations obtained formally by taking their limit when the Reynolds number goes to infinity are of first order. A singular perturbation problem arises.

\section{Direct analysis}

To go further, it is usual to consider small wall perturbations leading to hypothesis (H1):

(H1) $\left\{\begin{array}{l}F=\varepsilon f, \\ G=\varepsilon g .\end{array}\right.$

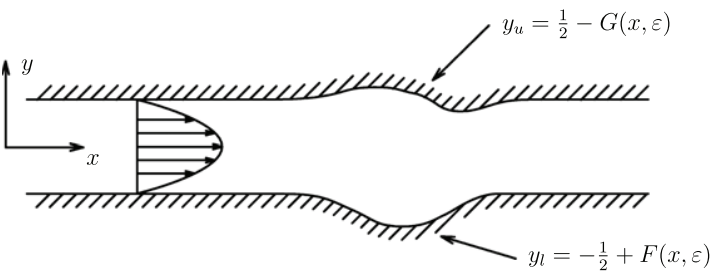

Fig. 1. Flow in a two-dimensional channel with deformed walls. In this figure, all quantities are dimensionless.

From the application of the standard method of matched asymptotic expansions, two zones are considered in the flow. One of them is the core where the flow perturbations are governed by Euler equations. The other zone is the proximity of walls where the flow perturbations develop in a structure of boundary layer type. A perturbation is said to be significant when flow separation is possible. To translate this, it is required that, in the boundary layers of thickness $\varepsilon$, the perturbation of the longitudinal velocity is of the same order as $u_{0}$, i.e., of order $\mathrm{O}(\varepsilon)$. Thus, according to the SCEM, we are seeking a UVA of the form

$u=u_{0}(y)+\varepsilon \hat{u}(x, y, \varepsilon)+\cdots$,

$\mathcal{V}=\varepsilon \hat{v}(x, y, \varepsilon)+\cdots$,

$\mathcal{P}-p_{c}=-\frac{2 x}{\mathcal{R}}+\varepsilon \hat{p}(x, y, \varepsilon)+\cdots$

For large Reynolds numbers, the core flow equations are [1]

$\frac{\partial \hat{u}_{1}}{\partial x}+\frac{\partial \hat{v}_{1}}{\partial y}=0$

$u_{0} \frac{\partial \hat{u}_{1}}{\partial x}+\hat{v}_{1} \frac{\mathrm{d} u_{0}}{\mathrm{~d} y}+\varepsilon\left(\hat{u}_{1} \frac{\partial \hat{u}_{1}}{\partial x}+\hat{v}_{1} \frac{\partial \hat{u}_{1}}{\partial y}\right)=-\frac{\partial \hat{p}_{1}}{\partial x}$,

$u_{0} \frac{\partial \hat{v}_{1}}{\partial x}+\varepsilon\left(\hat{u}_{1} \frac{\partial \hat{v}_{1}}{\partial x}+\hat{v}_{1} \frac{\partial \hat{v}_{1}}{\partial y}\right)=-\frac{\partial \hat{p}_{1}}{\partial y}$,

where the index " 1 " denotes the characteristics of the flow perturbation in the core. This means that (19)-(21) are valid only in the core.

In the whole flow field, the Navier-Stokes equations reduce to [1]

$\frac{\partial \hat{u}}{\partial x}+\frac{\partial \hat{v}}{\partial y}=0$

$u_{0} \frac{\partial \hat{u}}{\partial x}+\hat{v} \frac{\mathrm{d} u_{0}}{\mathrm{~d} y}+\varepsilon\left(\hat{u} \frac{\partial \hat{u}}{\partial x}+\hat{v} \frac{\partial \hat{u}}{\partial y}\right)=-\frac{\partial \hat{p}_{1}}{\partial x}+\frac{1}{\mathcal{R}} \frac{\partial^{2} \hat{u}}{\partial y^{2}}$.

As shown in $[1,4]$, it must be noted that in the streamwise momentum equation, $\frac{\partial \hat{p}}{\partial x}$ is replaced by $\frac{\partial \hat{p}_{1}}{\partial x}$. This means that the pressure required to solve (22)-(23) satisfies the core flow equations (19)-(21) but (22)-(23) are valid everywhere in the channel and they provide us with a UVA. Since this is the key point of the method, let us recall that the variables of the boundary layer are given by

$Y=\frac{\frac{1}{2}+y}{\varepsilon}, \quad \widehat{Y}=\frac{\frac{1}{2}-y}{\varepsilon}$.

It can be shown that a uniformly valid approximation of the pressure is given by

$\hat{p}(x, y, \varepsilon)=\hat{p}_{1}(x, y, \varepsilon)+\varepsilon^{3} \widehat{P}_{C L}(x, \hat{Y}, \varepsilon)+\varepsilon^{3} P_{C L}(x, Y, \varepsilon)$,

where $\widehat{P}_{C L}$ and $P_{C L}$ are zero in the core flow.

Eqs. (22)-(23) look like the standard boundary layer equations, but they are valid in the whole domain. To calculate the flow, it is 
required to solve the system comprising the generalized boundary layer equations (22)-(23) and the core flow equations (19)-(21). It is also necessary to prescribe the wall conditions

$y=y_{\ell} \quad$ and $y=y_{u}: u_{0}+\varepsilon \hat{u}=0, \quad \hat{v}=0$,

and to express the coupling between the core flow equations and the generalized boundary layer equations by identifying $\hat{u}, \hat{v}$ and $\hat{u}_{1}, \hat{v}_{1}$ in the core:

$(\hat{u}, \hat{v}) \rightarrow\left(\hat{u}_{1}, \hat{v}_{1}\right)$.

The global interactive boundary layer model described by (19)-(21), (22)-(23), (26), (27) is the best approximation of the Navier-Stokes model we can propose.

In $[1,13]$, it is seen that the boundary layer analysis leads to

$\mathcal{R}=\varepsilon^{-3}$.

Then, using the Navier-Stokes operator defined in (9)-(10), we observe that

$L_{\varepsilon} \hat{u}=\frac{\partial p}{\partial x}-\frac{\partial \hat{p}_{1}}{\partial x}-\frac{1}{\mathcal{R}} \frac{\partial^{2} \hat{u}}{\partial x^{2}}, \quad L_{\varepsilon} \hat{v}=-\frac{1}{\mathcal{R}} \frac{\partial^{2} \hat{v}}{\partial x^{2}}$.

From (25) and (28), it is easily shown that

$L_{\varepsilon} \hat{u}=\mathrm{O}\left(\varepsilon^{3}\right), \quad L_{\varepsilon} \hat{v}=\mathrm{O}\left(\varepsilon^{3}\right)$,

which shows, as stated before, that this is a very good approximation.

The nonlinear core equations are not easy to solve. Fortunately, as shown below from a deeper analysis, the core flow equations can be linearized, and the solution of the resulting model is much easier.

In the boundary layer whose thickness is of order $\varepsilon, u_{0}=\mathrm{O}(\varepsilon)$, $\hat{u}=\mathrm{O}(1)$ and $\hat{v}=\mathrm{O}(\varepsilon)$, so the three terms

$\left(u_{0} \frac{\partial \hat{u}}{\partial x}+\hat{v} \frac{\mathrm{d} u_{0}}{\mathrm{~d} y}\right), \quad \varepsilon\left(\hat{u} \frac{\partial \hat{u}}{\partial x}+\hat{v} \frac{\partial \hat{u}}{\partial y}\right), \quad \varepsilon^{3} \frac{\partial^{2} \hat{u}}{\partial y^{2}}$,

are of the same order, $\mathrm{O}(\varepsilon)$

In the core of the flow, the orders of magnitude are not the same. The terms $\hat{u}_{1}, \frac{\partial \hat{u}_{1}}{\partial x}, \frac{\partial \hat{u}_{1}}{\partial y}, \hat{v}_{1}, \frac{\partial \hat{v}_{1}}{\partial x}, \frac{\partial \hat{v}_{1}}{\partial y}$ are all O(1). Therefore, the field equations are structurally nonlinear, whereas the core flow Euler equations can be linearized rightfully.

If we define

$u=u_{0}+\varepsilon \hat{u}$,

$v=\varepsilon \hat{v}$,

and

$u_{1}=u_{0}+\varepsilon \hat{u}_{1}$,

$v_{1}=\varepsilon \hat{v}_{1}$,

$p_{1}=p_{0}+\varepsilon \hat{p}_{1}$,

the field and core flow equations become

$\frac{\partial u}{\partial x}+\frac{\partial v}{\partial y}=0$

$u \frac{\partial u}{\partial x}+v \frac{\partial u}{\partial y}=-\frac{\partial p_{1}}{\partial x}+\frac{1}{\mathcal{R}} \frac{\partial^{2} u}{\partial y^{2}}$,

$\frac{\partial u_{1}}{\partial x}+\frac{\partial v_{1}}{\partial y}=0$

$u_{0} \frac{\partial u_{1}}{\partial x}+v_{1} \frac{\mathrm{d} u_{0}}{\mathrm{~d} y}=-\frac{\partial}{\partial x}\left(p_{1}-p_{0}\right)$

$u_{0} \frac{\partial v_{1}}{\partial x}=-\frac{\partial}{\partial y}\left(p_{1}-p_{0}\right)$.
It is interesting to note the singular behaviour of the solution of (37)-(39) in the neighbourhood of the boundaries. For instance, as $y \rightarrow-1 / 2$, we have

$u_{1}-u_{0}=-2 p_{10} \ln \left|\frac{1}{2}+y\right|+c_{10}+\cdots$,

where $p_{10}$ and $c_{10}$ are functions of $x$ and $\varepsilon$.

The logarithmic singularity of this solution near $y=-1 / 2$ and $y=1 / 2$ exhibits an essential difference compared with external boundary layers. In the following, for this reason, the evolution of the perturbed flow in the core will be characterized by $v_{1}$. From (37)-(39), it is found that $v_{1}$ is a solution of Poisson's equation:

$u_{0}\left(\frac{\partial^{2} v_{1}}{\partial x^{2}}+\frac{\partial^{2} v_{1}}{\partial y^{2}}\right)=v_{1} \frac{\mathrm{d}^{2} u_{0}}{\mathrm{~d} y^{2}}$,

and the $x$-component of the pressure gradient required to solve the generalized boundary layer equations is given by (38), in which the continuity equation (37) is taken into account:

$-u_{0} \frac{\partial v_{1}}{\partial y}+v_{1} \frac{\mathrm{d} u_{0}}{\mathrm{~d} y}=-\frac{\partial}{\partial x}\left(p_{1}-p_{0}\right)$.

It can be shown that (41) associated to (42) gives the $y$ momentum equation (39) if the perturbations vanish at upstream infinity. This establishes the equivalence between (37)-(39) and (41)-(42).

To sum up, the problem to solve comprises (35)-(36), (41) and (42). At the walls, the boundary conditions are

$y=y_{\ell}$ and $y=y_{u}: u=0, \quad v=0$,

and the coupling between the core flow equations and the generalized boundary layer equations is expressed by identifying $u, v$ and $u_{1}, v_{1}$ in the core:

$(u, v) \rightarrow\left(u_{1}, v_{1}\right)$.

The model presented above belongs to the class of strong coupling models since there is no hierarchy between the boundary layer equations and the core flow equations. The triple deck theory, or more precisely its equivalent for channel flows as developed by Smith $[7,8]$, also belongs to this class of strong coupling models. In fact, Smith's model is included in the present model since the expansions are regular, whereas in the present model the expansions are generalized. It is interesting to note that the first approximation of Smith's model for $v_{1}$ is symmetric with respect to $y$ and corresponds to a geometrically antisymmetric problem. This point will be discussed later when a regular analysis is developed (Section 6). In the core, Smith's model gives

$v_{1}=-u_{0}(y) \frac{\mathrm{d} A(x)}{\mathrm{d} x}$,

where $A$ is defined as the displacement function.

It must be noted that (45) is an eigensolution of (42) but not of (41). In addition, the approximation (45) does not have the singular behaviour of (40). These two remarks lead us to try to separate as far as possible the symmetric and antisymmetric problems, which leads, as we will see, to a new approach of the asymptotic problem.

\section{Influence of asymmetry}

The analysis starts from Navier-Stokes equations (8)-(10) in which we introduce the transformation

$X=x$,

$Y=y-H(x, \varepsilon)$,

$U=U$,

$V=\mathcal{V}-u \frac{\mathrm{d} H}{\mathrm{~d} x}$,

$P=\mathcal{P}$, 
where $\varepsilon$ is formally the thickness of the boundary layer, i.e., the region where the inertial terms are of the same order as the viscous terms.

This point is also important in transferring the perturbations from the walls into the equations. The asymptotic analysis is also easier to do.

In order to define $H$, we set

$E=\frac{F+G}{2}$,

$H=\frac{F-G}{2}$,

so that the problem is geometrically symmetrized in the transformed plane. Obviously, the solution is not symmetrical. Note also that the physical channel is deformed symmetrically when $H=0$. In general, the wall conditions are

$Y=Y_{\ell}=-\frac{1}{2}+E$ and $Y=Y_{u}=\frac{1}{2}-E: U=0 ; \quad V=0$.

We introduce hypothesis (H2):

$(H 2): E=\mathrm{O}(\varepsilon), \quad v \mu^{2} \preceq \varepsilon$,

with

$H=v h(\mu x)$,

where $\nu(\varepsilon)$ and $\mu(\varepsilon)$ are order functions.

This extension of hypothesis (H1) shows that actually hypothesis (H1) applies only on the symmetric part of the perturbation.

With these hypotheses, the Navier-Stokes equations become

$\frac{\partial U}{\partial X}+\frac{\partial V}{\partial Y}=0$

$U \frac{\partial U}{\partial X}+V \frac{\partial U}{\partial Y}=-\frac{\partial P}{\partial X}+\frac{1}{\mathcal{R}}\left(\frac{\partial^{2} U}{\partial X^{2}}+\frac{\partial^{2} U}{\partial Y^{2}}\right)+O(v \mu)$,

$U \frac{\partial V}{\partial X}+V \frac{\partial V}{\partial Y}=-\frac{\partial P}{\partial Y}+\frac{1}{\mathcal{R}}\left(\frac{\partial^{2} V}{\partial X^{2}}+\frac{\partial^{2} V}{\partial Y^{2}}\right)+O(v \mu)$.

For small $\varepsilon$, the basic flow corresponding to $E=0$ is

$U_{0}=\frac{1}{4}-Y^{2}$,

$V_{0}=0$,

$P_{0}=-2 \frac{X}{\mathcal{R}}+P_{c}$

where $P_{c}$ is an arbitrary constant.

The Navier-Stokes equations can now be written as

$\frac{\partial U}{\partial X}+\frac{\partial V}{\partial Y}=0$

$U \frac{\partial U}{\partial X}+V \frac{\partial U}{\partial Y}=-\frac{\partial P}{\partial X}+\frac{\mathrm{d} H}{\mathrm{~d} X} \frac{\partial P}{\partial Y}+\frac{1}{\mathcal{R}} \frac{\partial^{2} U}{\partial Y^{2}}+\mathrm{O}\left(\frac{1}{\mathcal{R}}\right)$,

$U \frac{\partial V}{\partial X}+V \frac{\partial V}{\partial Y}+\frac{\mathrm{d}^{2} H}{\mathrm{~d} X^{2}} U^{2}=-\left[1+\left(\frac{\mathrm{d} H}{\mathrm{~d} X}\right)^{2}\right] \frac{\partial P}{\partial Y}+\frac{\mathrm{d} H}{\mathrm{~d} X} \frac{\partial P}{\partial X}$

$+\frac{1}{\mathcal{R}} \frac{\partial^{2} V}{\partial Y^{2}}+\mathrm{O}\left(\frac{1}{\mathcal{R}}\right)$

For the core flow whose characteristics are denoted with index "1", the Euler equations become $\frac{\partial U_{1}}{\partial X}+\frac{\partial V_{1}}{\partial Y}=0$

$U_{1} \frac{\partial U_{1}}{\partial X}+V_{1} \frac{\partial U_{1}}{\partial Y}=-\frac{\partial}{\partial X}\left(P_{1}-P_{0}\right)+\frac{\mathrm{d} H}{\mathrm{~d} X} \frac{\partial}{\partial Y}\left(P_{1}-P_{0}\right)$,

$U_{1} \frac{\partial V_{1}}{\partial X}+V_{1} \frac{\partial V_{1}}{\partial Y}+\frac{\mathrm{d}^{2} H}{\mathrm{~d} X^{2}} U_{1}^{2}=-\left[1+\left(\frac{\mathrm{d} H}{\mathrm{~d} X}\right)^{2}\right] \frac{\partial}{\partial Y}\left(P_{1}-P_{0}\right)$

$+\frac{\mathrm{d} H}{\mathrm{~d} X} \frac{\partial}{\partial X}\left(P_{1}-P_{0}\right)$.

As in (32)-(34), the flow characteristics can be expanded about the basic flow characteristics (58)-(60). Then, the following orders of magnitude are found:

$U_{1}=\mathrm{O}_{\mathrm{S}}(1), \quad \frac{\partial U_{1}}{\partial X}=\mathrm{O}(\varepsilon), \quad \frac{\partial U_{1}}{\partial Y}=\mathrm{O}_{\mathrm{S}}(1)$,

$V_{1}=\mathrm{O}(\varepsilon), \quad \frac{\partial V_{1}}{\partial X}=\mathrm{O}(\varepsilon), \quad \frac{\partial V_{1}}{\partial Y}=\mathrm{O}(\varepsilon)$.

It is recalled that the symbol $\mathrm{O}_{S}$ means "is of strict order of" whereas the symbol 0 means "is at most of order of" [1].

With hypothesis ( $\mathrm{H} 2)$, the left-hand sides of the second and third equations of (64) are of order $\varepsilon$. It is deduced that

$\frac{\partial}{\partial X}\left(P_{1}-P_{0}\right)=\mathrm{O}(\varepsilon), \quad \frac{\partial}{\partial Y}\left(P_{1}-P_{0}\right)=\mathrm{O}(\varepsilon)$.

As in Section 4, this leads us to the linearized core flow equations:

$\frac{\partial U_{1}}{\partial X}+\frac{\partial V_{1}}{\partial Y}=0$

$U_{0} \frac{\partial U_{1}}{\partial X}+V_{1} \frac{\mathrm{d} U_{0}}{\mathrm{~d} Y}=-\frac{\partial}{\partial X}\left(P_{1}-P_{0}\right)$,

$U_{0} \frac{\partial V_{1}}{\partial X}+\frac{\mathrm{d}^{2} H}{\mathrm{~d} X^{2}} U_{0}^{2}=-\frac{\partial}{\partial Y}\left(P_{1}-P_{0}\right)$.

Moreover, for large Reynolds numbers, as in [1], it can be shown that, in the boundary layer, $\frac{\partial P}{\partial X}$ can be replaced by $\frac{\partial P_{1}}{\partial X}$ in the streamwise momentum equation.

Finally, the complete system to solve comprises the core flow equations (66)-(68) and the field equations (61)-(62), simplified as

$\frac{\partial U}{\partial X}+\frac{\partial V}{\partial Y}=0$

$U \frac{\partial U}{\partial X}+V \frac{\partial U}{\partial Y}=-\frac{\partial P_{1}}{\partial X}+\frac{1}{\mathcal{R}} \frac{\partial^{2} U}{\partial Y^{2}}$

It must be noted that Eqs. (69)-(70) are valid not only in the boundary layers but everywhere in the channel.

The conditions required to solve this system are the boundary conditions (53) and the coupling condition in the core:

$V \rightarrow V_{1}$.

Since $\frac{\mathrm{d}^{2} H}{\mathrm{~d} X^{2}}$ is an approximation of the curvature along the median line $Y=H(X)$, the resulting problem obtained using the SCEM is analogous to the problem sets with hypothesis (H1), with an additional term in Eq. (68).

\section{Regular analysis of the transformed problem}

The idea is to perform a regular analysis of the model based on (69)-(70), (66)-(68), (71) and to show that this model contains in particular the first-order results obtained by Smith. A slow variable $\bar{X}$ is introduced:

$\bar{X}=X \varepsilon^{\alpha}$. 
By defining $\bar{V}$ such that

$V=\varepsilon^{\alpha} \bar{V}$

the continuity equation is

$\frac{\partial U}{\partial \bar{X}}+\frac{\partial \bar{V}}{\partial Y}=0$.

It is assumed that $\alpha \geq 0$ so that the length of the wall deformations is much larger than the channel width or is of the same strict order. The streamwise momentum equation (70) becomes

$U \frac{\partial U}{\partial \bar{X}}+\bar{V} \frac{\partial U}{\partial Y}=-\frac{\partial P_{1}}{\partial \bar{X}}+\frac{1}{R \varepsilon^{\alpha}} \frac{\partial^{2} U}{\partial Y^{2}}$.

It is easy to show that, in the vicinity of walls, the regular analysis of (75) leads to boundary layer equations which have the same form as (75).

Now, let us examine the core flow equations. By defining $\bar{V}_{1}$ such that

$\bar{V}_{1} \varepsilon^{\alpha}=V_{1}$,

the core flow equations (66)-(68) become

$-U_{0} \frac{\partial \bar{V}_{1}}{\partial Y}+\bar{V}_{1} \frac{\mathrm{d} U_{0}}{\mathrm{~d} Y}=-\frac{\partial}{\partial \bar{X}}\left(P_{1}-P_{0}\right)$,

$\varepsilon^{2 \alpha} U_{0} \frac{\partial \bar{V}_{1}}{\partial \bar{X}}+\frac{\mathrm{d}^{2} H}{\mathrm{~d} X^{2}} U_{0}^{2}=-\frac{\partial}{\partial Y}\left(P_{1}-P_{0}\right)$.

As we know the behaviour of the pressure, we look for regular expansions beginning with

$\bar{V}_{1}(\bar{X}, Y, \varepsilon)=\varepsilon^{r} \bar{V}_{10}(\bar{X}, Y)+\cdots$,

$P_{1}(\bar{X}, Y, \varepsilon)-P_{0}=\varepsilon^{2} P_{10}(\bar{X}, Y)+\cdots$.

With the above expansions, $\bar{V}_{1}=\mathrm{O}(\varepsilon)$ implies that $r \geq 1$. In addition, the regular analysis imposes $\mu=\varepsilon^{\alpha}$ and $H=\varepsilon h(\bar{X})$.

The analysis of (77)-(78) with the asymptotic expansions (79)-(80) leads us to consider two cases.

1. $\alpha>\frac{1}{2}$. To first order, the pressure is constant in a cross-section

$\frac{\partial P_{10}}{\partial Y}=0$,

and, for a direct comparison with Smith's results, the solution for $\bar{V}_{1}$ can be cast in the form

$\bar{V}_{1}=-\varepsilon U_{0}\left(\frac{\mathrm{d} A}{\mathrm{~d} \bar{X}}+\frac{\mathrm{d} h}{\mathrm{~d} \bar{X}}\right)$,

where $A(\bar{X})$ is a function of $\bar{X}$ known as the displacement function.

As the global problem to solve is fully symmetric with respect to $Y, \bar{V}_{10}$ must be antisymmetric, but (82) gives a symmetric solution if $A+h \neq 0$. Thus, we have to take $A+h=0$. To the considered order, the perturbation is negligible.

2. $\alpha \leq \frac{1}{2}$. The solution for $\bar{V}_{1}$ is

$\bar{V}_{1}=-\varepsilon \frac{\mathrm{d} h}{\mathrm{~d} \bar{X}} U_{0}(Y)-\varepsilon^{2-2 \alpha} \frac{\mathrm{d} A}{\mathrm{~d} \bar{X}} U_{0}(Y)+\cdots$,

and, for $\alpha<1 / 2$, the pressure variation in a cross-section is given by

$\frac{\partial P_{10}}{\partial Y}=\frac{\mathrm{d}^{2} A}{\mathrm{~d} \bar{X}^{2}} U_{0}^{2}(Y)$.

Here, the displacement function $A(\bar{X})$ must be determined from the matching condition between the core and the boundary layer.
For $\alpha=1 / 2$, we have

$$
\begin{aligned}
& \bar{V}_{1}=-\varepsilon\left[\frac{\mathrm{d} h}{\mathrm{~d} \bar{X}}+\frac{\mathrm{d} A}{\mathrm{~d} \bar{X}}\right] U_{0}+\cdots, \\
& \frac{\partial P_{10}}{\partial Y}=U_{0}^{2}(Y) \frac{\mathrm{d}^{2} A}{\mathrm{~d} \bar{X}^{2}} .
\end{aligned}
$$

Finally, the solution for $U_{1}, V_{1}$ is

1. $\alpha>\frac{1}{2}$

$$
\begin{aligned}
& U_{1}=U_{0}(Y)+\mathrm{O}\left(\varepsilon^{2}\right), \\
& V_{1}=\mathrm{O}\left(\varepsilon^{2}\right) .
\end{aligned}
$$

This case is interesting because it gives the limits of a problem where the walls are not exactly symmetric but which can be treated as being symmetric.

2. $\alpha \leq \frac{1}{2}$

$$
\begin{aligned}
& U_{1}=U_{0}(Y)+\varepsilon h(\bar{X}) \frac{\mathrm{d} U_{0}}{\mathrm{~d} Y}+\varepsilon^{2-2 \alpha} A(\bar{X}) \frac{\mathrm{d} U_{0}}{\mathrm{~d} Y}+\cdots, \\
& V_{1}=-\varepsilon^{1+\alpha} \frac{\mathrm{d} h}{\mathrm{~d} \bar{X}} U_{0}(Y)-\varepsilon^{2-\alpha} \frac{\mathrm{d} A}{\mathrm{~d} \bar{X}} U_{0}(Y)+\cdots
\end{aligned}
$$

Let us recall that the regular form of the SCEM leads to a uniformly valid approximation to a given order, here $\varepsilon$. In addition, the results are equivalent to those obtained with the standard method of matched asymptotic expansions when the matching is performed to the same order according to the modified Van Dyke principle [1]. For $U$, we have formally

$U=U_{1}+\varepsilon U_{\mathrm{BL}}-\varepsilon U_{\mathrm{c}}$,

where $U$ is a UVA to order $\varepsilon, U_{1}$ is the approximation in the core to order $\varepsilon$, and $\varepsilon U_{\mathrm{BL}}$ is the approximation in the boundary layer to order $\varepsilon$. The quantity $U_{\mathrm{c}}$ results from the matching condition to order $\varepsilon$. For example, in the vicinity of $Y=1 / 2$, the boundary layer variable is

$\widetilde{Y}=\frac{\frac{1}{2}-Y}{\varepsilon}$.

With

$U_{0}=\frac{1}{4}-Y^{2}$,

we obtain the following matching conditions:

1. $\alpha<\frac{1}{2}: \lim _{\widetilde{Y} \rightarrow \infty}\left(U_{\mathrm{BL}}-\tilde{Y}\right)=-h(\bar{X})$,

2. $\alpha=\frac{1}{2}: \lim _{\widetilde{Y} \rightarrow \infty}\left(U_{\mathrm{BL}}-\tilde{Y}\right)=-h(\bar{X})-A(\bar{X})$,

3. $\alpha>\frac{1}{2}: \lim _{\widetilde{Y} \rightarrow \infty}\left(U_{\mathrm{BL}}-\widetilde{Y}\right)=0$.

These results can be interpreted in terms of the original variables. Taking into account the transformation (47), and defining the boundary layer variable $\tilde{y}=\left(\frac{1}{2}-y\right) / \varepsilon$, we have

$\tilde{Y}=\tilde{y}+h(\bar{X})$,

so the matching conditions become

1. $\alpha<\frac{1}{2}: \lim _{\tilde{y} \rightarrow \infty}\left(u_{\mathrm{BL}}-\tilde{y}\right)=0$,

2. $\alpha=\frac{1}{2}: \lim _{\tilde{y} \rightarrow \infty}\left(u_{\mathrm{BL}}-\tilde{y}\right)=-A(\bar{X})$,

3. $\alpha>\frac{1}{2}: \lim _{\tilde{y} \rightarrow \infty}\left(u_{\mathrm{BL}}-\tilde{y}\right)=h(\bar{X})$.

If all the results obtained in this section with the transformed variables are interpreted in terms of the original variables, they are identical to those obtained in $[1,7,8]$. This means that the model based on (69)-(70), (66)-(68), (71) contains in particular the model developed by Smith to first order, but is more general. 


\section{Numerical procedure}

According to the analysis proposed in Section 5, the problem is to solve the generalized boundary layer equations (69)-(70) associated to the core flow equations (66)-(68). The velocity components $U$ and $V$ vanish at the walls, and the coupling condition between the boundary layer solution and the core flow solution is given by (71).

First, the core flow equations are rewritten by introducing a change of variable:

$V_{1}^{*}=V_{1}+\frac{\mathrm{d} H}{\mathrm{~d} X} U_{0}$.

Then, it is shown that $V_{1}^{*}$ obeys Poisson's equation:

$U_{0} \frac{\partial^{2} V_{1}^{*}}{\partial X^{2}}+U_{0} \frac{\partial^{2} V_{1}^{*}}{\partial Y^{2}}=V_{1}^{*} \frac{\mathrm{d}^{2} U_{0}}{\mathrm{~d} Y^{2}}$.

The coupling condition becomes

$V=V_{1}^{*}-\frac{\mathrm{d} H}{\mathrm{~d} X} U_{0}$.

To solve the equations, a change of coordinates is introduced,

$\xi=X$

$\eta=\frac{Y}{\zeta(X)} \quad$ with $\zeta(X)=1-2 E(X)$,

so that the domain is limited by the lines $\eta=-1 / 2$ and $\eta=1 / 2$ which define the channel walls.

In agreement with the analysis of Section 5, the core flow equations are linearized in the $(\xi, \eta)$-plane, so that Poisson's equation for $V_{1}^{*}$ keeps the same form as (95). In this equation, the velocity of the basic flow is

$U_{0}^{*}=\frac{1}{4}-\eta^{2}$.

The generalized boundary layer equations are rewritten by introducing the following velocity transformation:

$U^{*}=U$,

$V^{*}=V-U \eta \frac{\mathrm{d} \zeta}{\mathrm{d} X}$,

so the generalized boundary layer equations are only slightly modified.

The numerical procedure used to solve the whole set of equations is discussed below.

Poisson's equation for $V_{1}^{*}$ is discretized using a five-point stencil. A successive line overrelaxation method is applied to solve the resulting equations. According to this technique, an iterative procedure is implemented in which the calculation domain is swept from upstream to downstream. When the solution is calculated at a given $\xi$-station, the value of $V_{1}^{*}$ at the upstream $\xi$-station is the updated value of $V_{1}^{*}$ calculated at the current iteration, whereas the value of $V_{1}^{*}$ at the downstream station is taken at the previous iteration. The sweeping of the calculation domain is repeated until convergence is achieved.

At a given $\xi$-station, the longitudinal component of the pressure gradient is calculated from the core flow streamwise momentum equation and is used to solve the generalized boundary layer equations. These equations are discretized using a standard finite difference method with three points in the $\eta$-direction and two points in the $\xi$-direction.

In fact, Poisson's equation and the generalized boundary layer equations are strongly coupled, and the whole set of equations is solved as simultaneously as possible. The procedure is described below.

The calculation of the values of $U^{*}, V^{*}$ and $V_{1}^{*}$ at the different grid points for a given $\xi$-station consists of solving a system of algebraic equations in which the coefficients of the unknowns form tridiagonal matrices. The main problem is that the values of $V_{1}^{*}$ are not known a priori along the lines $\eta=-1 / 2$ and $\eta=1 / 2$.

At a given $\xi$-station, the solution of Poisson's equation can be written as

$V_{1 i}^{*}=A_{i} V_{1}^{*}\left(-\frac{1}{2}\right)+B_{i} V_{1}^{*}\left(\frac{1}{2}\right)+C_{i}$,

where $V_{1 i}^{*}$ is the value of $V_{1}^{*}$ at a certain grid point $i$ along a line $\xi=$ const. The coefficients $A_{i}, B_{i}$ and $C_{i}$ do not depend on the values $V_{1}^{*}(-1 / 2)$ and $V_{1}^{*}(1 / 2)$, and are calculated explicitly.

In the same way, after replacing the streamwise component of the pressure gradient by its expression as a function of $V_{1}^{*}$ and of its transverse derivative, the solution of the generalized boundary layer equation for $U^{*}$ has the form

$U_{i}^{*}=a_{i} V_{1}^{*}\left(-\frac{1}{2}\right)+b_{i} V_{1}^{*}\left(\frac{1}{2}\right)+c_{i}$,

and for $V^{*}$, we have

$V_{i}^{*}=\alpha_{i} V_{1}^{*}\left(-\frac{1}{2}\right)+\beta_{i} V_{1}^{*}\left(\frac{1}{2}\right)+\gamma_{i}$.

Solution (103) for $U^{*}$ is obtained by applying the wall conditions on $U^{*}$. This means that the coefficients $a_{i}, b_{i}$ and $c_{i}$ are calculated by taking into account these boundary conditions. For $V^{*}$, the above solution (104) is obtained by using the condition $V^{*}=0$ along one wall only, for example the lower wall. Indeed, only one boundary condition can be taken into account because $V^{*}$ is deduced from $U^{*}$ by integrating the continuity equation which is of first order with respect to the transverse coordinate.

At this stage, two conditions are not yet prescribed. The first condition is $V^{*}=0$ along the other wall (the upper wall); this condition is equivalent to the prescription of a constant mass flow in the whole channel. The second condition is the coupling between the boundary layer solution and the core flow solution. These two missing conditions are written from the expressions of $V_{1 i}^{*}, U_{i}^{*}$ and $V_{i}^{*}$. For example, the coupling condition is

$$
\begin{aligned}
& A_{c} V_{1}^{*}\left(-\frac{1}{2}\right)+B_{c} V_{1}^{*}\left(\frac{1}{2}\right)+C_{c}-\frac{\mathrm{d} H}{\mathrm{~d} \xi} U_{0}^{*}\left(\eta_{c}\right) \\
& =\alpha_{c} V_{1^{*}}\left(-\frac{1}{2}\right)+\beta_{c} V_{1}^{*}\left(\frac{1}{2}\right)+\gamma_{c}+U_{c}^{*} \eta_{c} \frac{\mathrm{d} \zeta}{\mathrm{d} \xi},
\end{aligned}
$$

where the index $c$ denotes the given core line along which the coupling conditions hold. Eq. (105) gives the first relation to calculate the values of $V_{1}^{*}(-1 / 2)$ and $V_{1}^{*}(1 / 2)$. The second relation is obtained by calculating the mass flow from the expression of $U_{i}^{*}$ (103).

When the values of $V_{1}^{*}(-1 / 2)$ and $V_{1}^{*}(1 / 2)$ are determined, the solutions for $V_{1 i}^{*}, U_{i}^{*}$ and $V_{i}^{*}$ are known from (102)-(104) and the calculation can proceed to the next $\xi$-station. In this way, the whole domain is swept from upstream to downstream. The sweep is repeated until convergence is achieved.

This procedure ensures a quasi-simultaneous resolution of the whole system of equations and makes the algorithm very efficient [14].

\section{Results}

To assess the validity of the interactive boundary layer (IBL) method, we examined the evolution of the skin-friction coefficient which is a very sensitive flow feature,

$C_{f}=\frac{2}{\mathcal{R}} \tau_{w}$,

where $\tau_{w}$ is the reduced wall shear stress. 

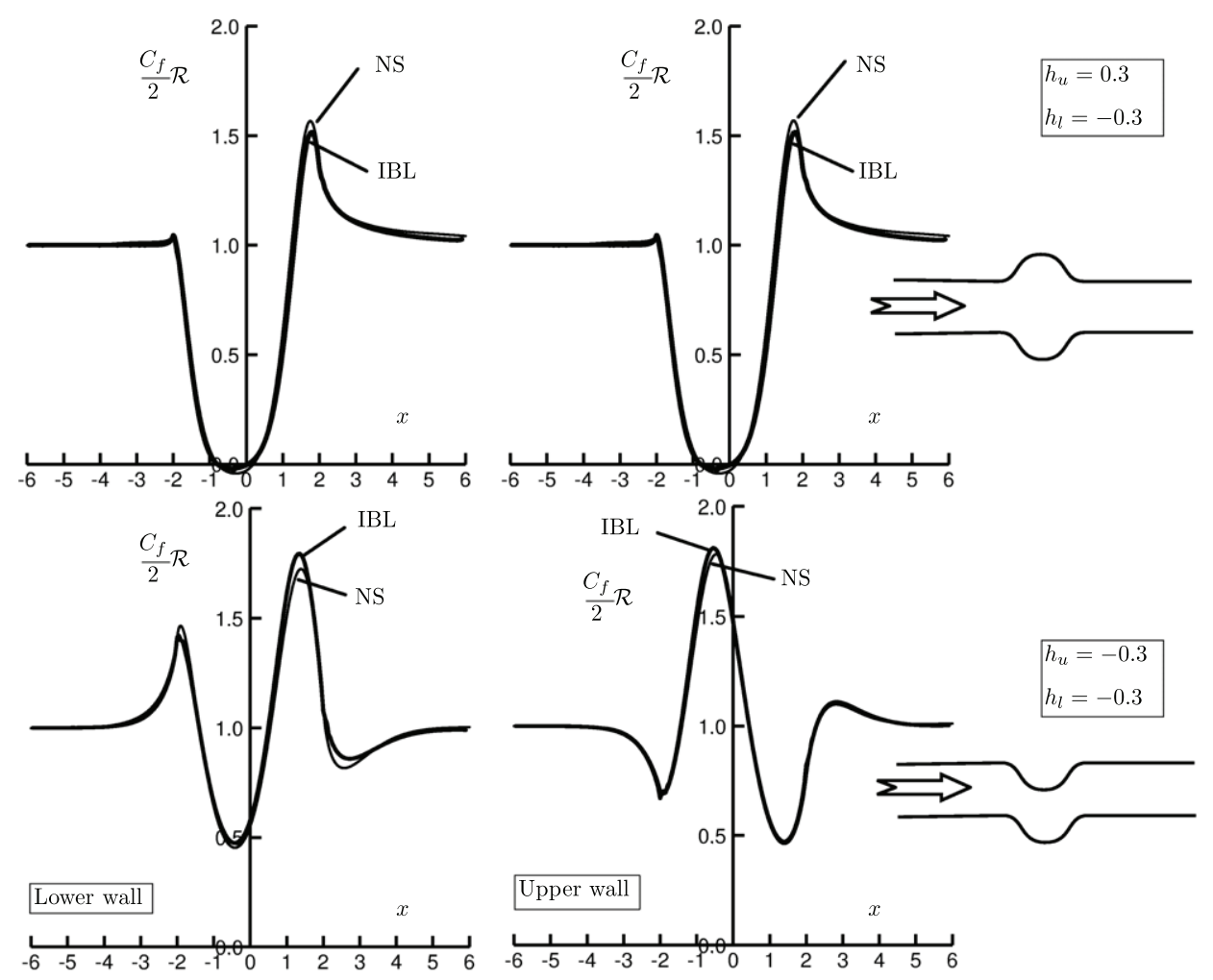

Fig. 2. Flow produced by symmetric and antisymmetric wall deformations. $\mathcal{R}=1000$. NS $=$ Navier-Stokes results, IBL $=$ interactive boundary layer results.

The Navier-Stokes calculations were performed with the commercial CFD package FLUENT. The test-case geometry is a channel whose walls are deformed in a domain $x_{1} \leq x \leq x_{2}$ :

$F=\frac{h_{l}}{2}\left(1+\cos \frac{2 \pi x}{L}\right) ; \quad G=-\frac{h_{u}}{2}\left(1+\cos \frac{2 \pi x}{L}\right)$.

For all cases, the Reynolds number is $\mathcal{R}=1000$.

First, comparisons between the IBL and Navier-Stokes results are given for the purely symmetric and antisymmetric wall deformations in Fig. 2. The lower wall is deformed by a trough located in the domain $-2 \leq x \leq 2(L=4)$ with $h_{l}=-0.3$. The upper wall is deformed in the same domain $-2 \leq x \leq 2$ $(L=4)$ with $h_{u}=0.3$ for the symmetric case and $h_{u}=-0.3$ for the antisymmetric case. Even though the amplitude of the wall deformation is not really small as required by the formal theory, an excellent agreement with the Navier-Stokes results is observed.

It can be noted that there is a very slight upstream influence in the symmetric case. In fact, the pressure is also nearly constant in a cross-section. This is not the case for antisymmetric geometries where the upstream influence is clearly evidenced.

The IBL model enables us also to treat original problems. In the case of a bend, when the channel does not recover its initial axis location at the downstream end, the usual techniques of small perturbations do not work any longer. As an example, the walls are deformed in the domain $-2 \leq x \leq 0(L=4)$ with $h_{l}=0.5$ and $h_{u}=0.5$; for $x>0$, we have $y_{l}=0, y_{u}=1$, so the channel axis is displaced from $y=0$ upstream to $y=0.5$ downstream. In this case again, a good agreement with Navier-Stokes results is observed (Fig. 3).

In this latter case, if the flow is far from being separated, a slight constriction can be added in the bend to induce separation deliberately. Thus, the walls are deformed by an additional symmetric constriction given by (106) with $L=2$ in the domain $-3 \leq x \leq-1$ with $h_{l}=0.1$ and $h_{u}=-0.1$. The same configuration with $h_{l}=0.2$ and $h_{u}=-0.2$ has also been calculated. The results are given in Figs. 4 and 5.
Then, we can observe that an antisymmetric deformation is much less disturbing than a symmetric deformation of same amplitude except if the flow is excited by a small upstream perturbation.

Along the same lines, it should be possible to consider channels whose downstream cross-section is not equal to the upstream cross-section. However, it is possible to give an idea of the quality of the results obtained with the IBL model presented here. To show this, the calculations are performed with the transformation (97)-(98) and with the basic flow (99). For instance, Figs. 6 and 7 show the results obtained for a constricted channel and a dilated channel. The shape of these channels is defined from (106). The walls are deformed in the domain $-2 \leq x \leq 0$ with $L=4$. In the case of the constricted channel, we have $h_{l}=0.2$ and $h_{u}=-0.2$; in the downstream part $(x>0)$, we have $y_{l}=-0.3$ and $y_{u}=0.3$. In the case of the dilated channel, we have $h_{l}=-0.4$ and $h_{u}=0.4$; in the downstream part $(x>0)$, we have $y_{l}=-0.9$ and $y_{u}=0.9$.

A very good agreement with the Navier-Stokes results is obtained again, even if a significant separated zone is present in the case of the dilated channel.

\section{Conclusion}

The method presented in this paper enables us to consider significant deformations of the channel walls. A few representative cases have been selected and the skin-friction coefficients have been compared to Navier-Stokes solutions.

The skin-friction coefficient is a very sensitive flow feature. A very good agreement is observed in difficult cases which cannot be treated easily with other methods such as the method developed by Smith which is the counterpart of the triple deck theory for internal flows.

The idea of strong coupling between the linear core flow equations and the nonlinear uniformly valid equations is called here the interacting boundary layer, IBL, for simplicity. We use also GIBL, $G$ for global, since the equations are valid in the 

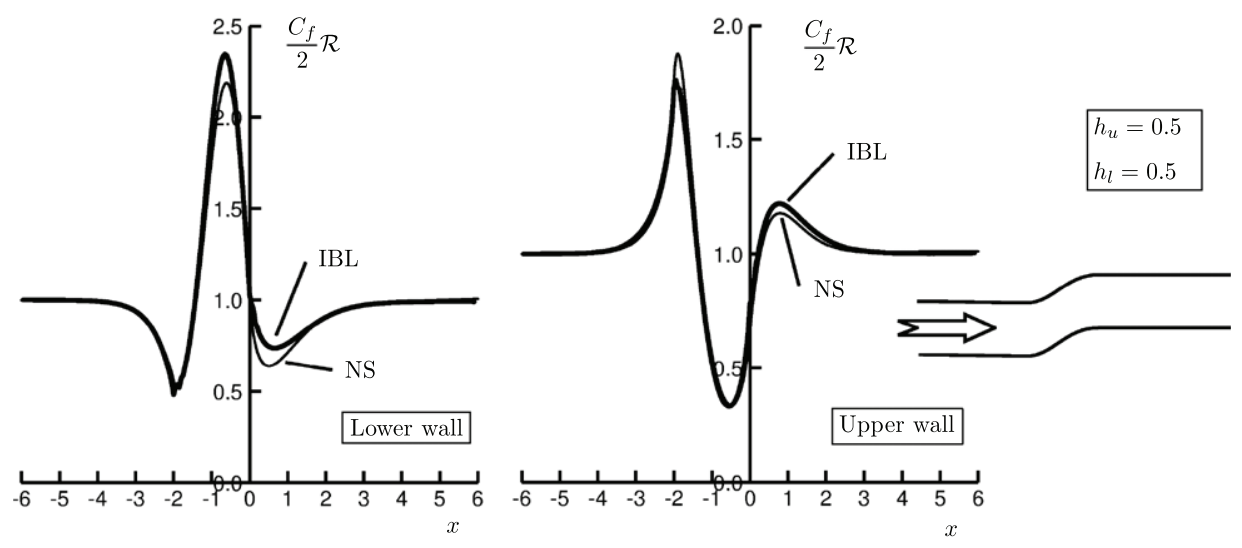

Fig. 3. Flow produced by a channel bend. $\mathcal{R}=1000$. NS $=$ Navier-Stokes results, $\mathrm{IBL}=$ interactive boundary layer results.
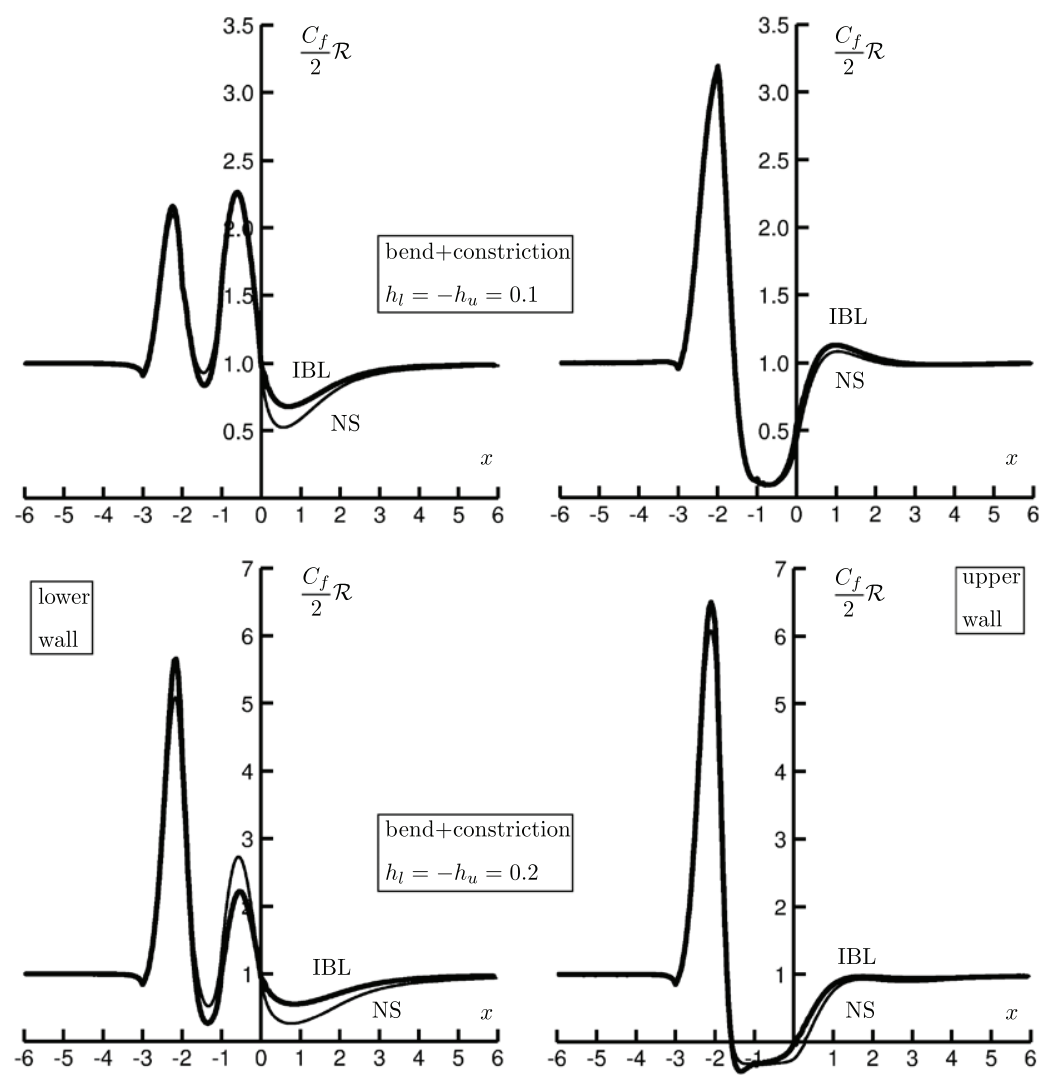

Fig. 4. Flow produced by a the superposition of a bend and of a symmetric constriction. $\mathcal{R}=1000$. NS $=$ Navier-Stokes results, IBL $=$ interactive boundary layer results.

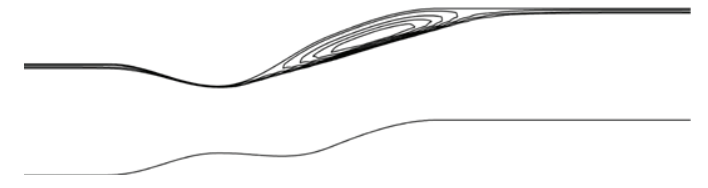

Fig. 5. Flow produced by the superposition of a bend and of a symmetric constriction. Streamlines calculated in the separated region with FLUENT.

whole domain. This approach is systematically used so that a non-hierarchical process is employed even if it is not necessary. Moreover, there is no matching and the boundary conditions are exactly satisfied.

The main goal of this study was to separate, as far as possible, the symmetrical problem and the antisymmetrical one. The final problem is geometrically symmetrized and the interaction with the antisymmetrical effects appears in the transverse Euler equation (68) through the second derivative of $H$, knowing that the problem is fully symmetrical for $H=0$.

Separation is more easily produced with a symmetrical dilatation. In that case, for $H=0$, numerical calculations show that $V_{1}=0$, and the transverse pressure variation is null which is a classical hypothesis and a good approximation. The drawback of taking a constant pressure in a cross-section is that this assumption prevents us from observing any upstream influence. However, in practice, this upstream influence is very slight.

In contrast, for the antisymmetrical case, the upstream influence is important as the transverse pressure gradient is not negligible. Moreover, when $E=0$, as $H$ can be of order 1, Poiseuille flow is only displaced so that the approximation (45) is only good locally. The analysis of the upstream interaction has been done in this framework by Smith [15]. 

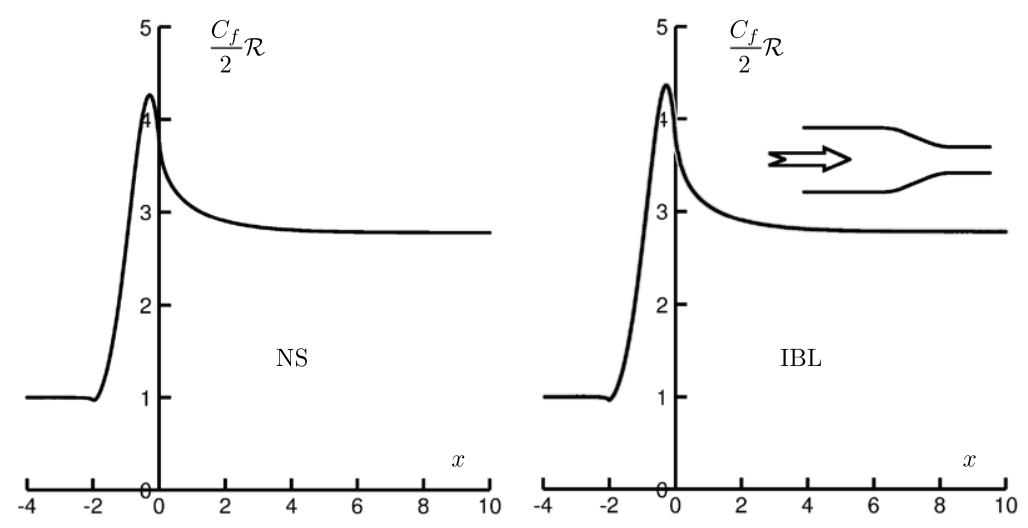

Fig. 6. Flow produced by a symmetric constriction. $\mathcal{R}=1000$. NS $=$ Navier-Stokes results, IBL $=$ interactive boundary layer results.
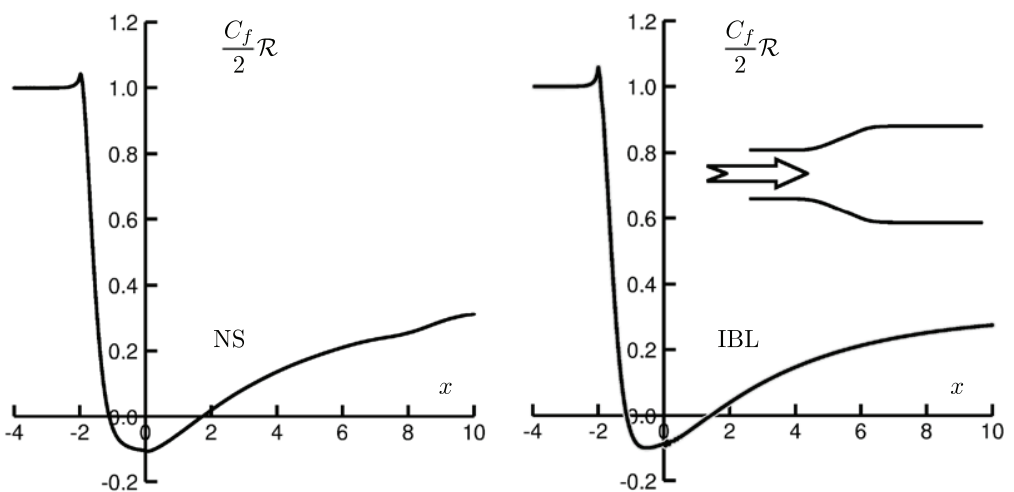

Fig. 7. Flow produced by a symmetric dilatation. $\mathcal{R}=1000$. NS $=$ Navier-Stokes results, $\mathrm{IBL}=$ interactive boundary layer results.

According to hypothesis (H2), within our degree of approximation, the second derivative of $H$ is the curvature of the curve $y=H(x)$. This suggests that it should be possible to consider channels whose downstream direction is no longer the direction of the $x$-axis, and thus is different from the upstream direction. In fact, the hypothesis that the first derivative of $H$ is small is not necessary. In the same way, in the numerical applications, we considered channels whose downstream cross-section is not equal to the upstream cross-section. Thus, it is possible to change the fact that $E$ is small. Obviously, the study of such cases will be considered in the frame of an extended theory with curvilinear coordinates, but interesting results have been obtained with the present approach.

This study confirms the soundness of the SCEM which, we recall, is based on the construction of a uniformly valid approximation. This is particularly fruitful in the case of strong coupling when, for example, boundary layer separation can occur, which implies a significant modification of the flow. The numerical calculations are faster for these simplified models than for Navier-Stokes solutions, but this is not the primary goal of the development of these models. In fact, this new asymptotic analysis, the so-called SCEM, helps us to understand the flow structure; this step was necessary for further important problems. It is clear that future developments are not lacking. If channel flows proved to be an interesting test to approach other problems such as flow ducts, it seems valuable to look at external flows and wakes with flow control, in laminar or turbulent regimes, which are a fundamental target and for which the SCEM seems to be particularly well adapted.

\section{References}

[1] J. Cousteix, J. Mauss, Asymptotic Analysis and Boundary Layers, in: Scientific Computation, vol. XVIII, Springer, Berlin, Heidelberg, 2007.

[2] I.J. Sobey, Introduction to Interactive Boundary Layer Theory, in: Oxford Applied and Engineering Mathematics, Oxford University Press, Oxford, 2000.

[3] F.T. Smith, On the high Reynolds number theory of laminar flows, IMA J. Appl. Math. 28 (3) (1982) 207-281.

[4] J. Cousteix, J. Mauss, Interactive boundary layer models for channel flow, Eur. J. Mech. B Fluids 28 (2009) 72-87.

[5] P.Y. Lagrée, E. Berger, M. Deverge, C. Vilain, A. Hirschberg, Characterization of the pressure drop in a 2D symmetrical pipe: Some asymptotical, numerical and experimental comparisons, ZAMM Z. Angew. Math. Mech. 85 (1) (2005) $1-6$

[6] P.Y. Lagrée, S. Lorthois, The RNS/Prandtl equations and their link with other asymptotic descriptions: Application to the wall shear stress in a constricted pipe, Internat. J. Engrg. Sci. 43 (2005) 352-378.

[7] F.T. Smith, Flow through constricted or dilated pipes and channels: Part 1 Quart. J. Mech. Appl. Math. XXIX (Pt 3) (1976) 343-364.

[8] F.T. Smith, Flow through constricted or dilated pipes and channels: Part 2 Quart. J. Mech. Appl. Math. XXIX (Pt 3) (1976) 365-376.

[9] W. Eckhaus, Matched Asymptotic Expansions and Singular Perturbations, North Holland, Amsterdam, London, 1973; American Elseiver, New York.

[10] R.E. O'Malley Jr., Introduction to singular perturbations, Appl. Math. Mech. 14 (1974).

[11] A.B. Vasil'eva, V.F. Butuzov, L.V. Kalachev, The boundary function method for singular perturbation problems, Stud. Appl. Math. 14 (1995)

[12] A.H. Nayfeh, Perturbations Methods, in: Pure and Applied Mathematics, John Wiley \& Sons, 1973

[13] J. Mauss, A. Dechaume, J. Cousteix, Global interactive boundary layer (GIBL) for a channel, Int. J. Comput. Sci. Math. 1 (2-4) (2007).

[14] A.E.P. Veldman, Matched asymptotic expansions and the numerical treatment of viscous-inviscid interaction, J. Engrg. Math. 39 (2001) 189-206.

[15] F.T. Smith, Upstream interactions in channel flows, J. Fluid Mech. 79 (4) (1977) 631-655. 